\title{
Rapid Symptom Improvement in Major Depressive Disorder Using Accelerated Repetitive Transcranial Magnetic Stimulation
}

\author{
Soo-Jeong Kim ${ }^{1,2}$, Sang Joon Son ${ }^{3}$, Mi Jang ${ }^{4}$, Byung-Hoon Kim ${ }^{1,2}$, Seok Joo Hong ${ }^{3}$, Lina Seo ${ }^{3}$, Sun-Woo Choi ${ }^{2}$, \\ Jeong-Ho Seok ${ }^{1,2}$, Jai Sung Noh $^{3}$ \\ ${ }^{1}$ Department of Psychiatry, Gangnam Severance Hospital, Yonsei University College of Medicine, ${ }^{2}$ Institute of Behavioral Science in Medicine, \\ Yonsei University College of Medicine, Seoul, ${ }^{3}$ Department of Psychiatry, Ajou University School of Medicine, Suwon, ${ }^{4}$ Department of Mental \\ Health Services, National Center for Mental Health, Seoul, Korea
}

\begin{abstract}
Objective: Repetitive transcranial magnetic stimulation (rTMS) has contributed to increase in the remission rate for patients with major depressive disorder (MDD). However, current rTMS treatment is practically inconvenient because it requires daily treatment sessions for several weeks. Accelerated rTMS treatment is as efficient and safe for MDD patients as conventional rTMS.

Methods: Fifty-one patients with MDD participated in this study; they were randomized into accelerated rTMS ( $\mathrm{n}=$ $21)$, conventional rTMS $(n=22)$, and sham-treatment $(n=8)$ groups. The accelerated and conventional rTMS groups received 15 sessions for 3 days and 3 weeks, respectively. The sham-treatment group received 15 sham rTMS sessions for 3 days. Primary outcome was assessed using self-report and clinician-rated Korean Quick Inventory of Depressive Symptomatology (KQIDS-SR and KQIDS-C, respectively). Adverse effects were monitored using the Frequency, Intensity, and Burden of Side Effects Rating scale. Changes in depressive symptoms were compared among the three groups using mixed model analyses.

Results: For the KQIDS-SR score, there was a significant main effect of "time" $\left(\mathrm{F}_{3,47}=11.05, p<0.001\right)$, but no effect of "group" $\left(\mathrm{F}_{2,47}=2.04, p=0.142\right)$, and a trend-level interaction effect of "group $\times$ time" $\left(\mathrm{F}_{6,47}=2.26, p=0.053\right)$. Improvement in depressive symptoms, based on the KQIDS-SR score 3 weeks after treatment, was more prominent in the accelerated rTMS group than in the sham-treatment group $(p=0.011)$. Tolerability was comparable among the three groups.

Conclusion: The accelerated rTMS treatment group showed rapid improvement of depressive symptoms compared with the sham-treatment and conventional rTMS treatment groups. Therefore, accelerated rTMS treatment could be a viable option for MDD, with improved accessibility.
\end{abstract}

KEY WORDS: Transcranial magnetic stimulation, repetitive; Major depressive disorder; Effectiveness, treatment; Safety; Accessibility, health services.

\section{INTRODUCTION}

Major depressive disorder (MDD) is a great burden to

Received: January 29, 2020 / Revised: February 28, 2020

Accepted: March 11, 2020

Address for correspondence: Jeong-Ho Seok

Department of Psychiatry, Gangnam Severance Hospital, Yonsei University College of Medicine, Room 507, 20 Eonju-ro 63-gil,

Gangnam-gu, Seoul 06229, Korea

E-mail: johnstein@yuhs.ac

ORCID: https://orcid.org/0000-0002-9402-7591

Jai Sung Noh

Department of Psychiatry, Ajou University, School of Medicine,

164 WorldCup-ro, Yeongtong-gu, Suwon 16499, Korea

E-mail: jsnoh@ajou.ac.kr

ORCID: https://orcid.org/0000-0002-9610-0756 society, with functional impairment of the patients and increasing suicidal risk. According to the World Health Organization, approximately 800,000 people commit suicide every year, and 9 out of 10 exhibit psychiatric illnesses, two-third of which is accounted for by depression, being the single largest leading cause [1]. Depression is also known to be the leading cause of ill health and disability [2]. The social and economic costs associated with depression are becoming a global burden [3-6].

Even though pharmacological treatment of MDD has been developed, a substantial proportion of the patients with depressive disorder remain partially treated or treatment-resistant. The Sequenced Treatment Alternatives to

(c) This is an Open-Access article distributed under the terms of the Creative Commons Attribution Non-Commercial License (http://creativecommons.org/licenses/by-nc/4.0) which permits unrestricted non-commercial use, distribution, and reproduction in any medium, provided the original work is properly cited. 
Relieve Depression study reported that $33 \%$ of the patients with MDD do not show remission after four trials of different antidepressant treatments, from monotherapy to combination or augmentation therapy [7]. There are other well-established treatment options for patients with depressive disorder, such as psychotherapy and cognitive behavior therapy. However, it is difficult for patients with MDD exhibiting severe depressive symptoms to participate in these types of treatment, which require high level of psychological or cognitive resources. Although electroconvulsive therapy is the oldest non-invasive brain stimulation for MDD patients, it requires anesthesia and potentially cause cognitive sequelae [8]. Recently, transcranial magnetic stimulation (rTMS) treatment is being emphasized as an alternative approach because it does not require anesthesia and cause little cognitive side-effects [9].

Treatment with rTMS may have the potential to increase the remission rate of patients with depression by inducing improvement in symptoms that do not respond to antidepressants or by providing incentive to patients with MDD who are reluctant to undergo medication to visit the clinic for treatment. Meta-analysis studies show that rTMS treatment is superior to sham treatment in improving depressive symptoms and inducing remission [10-12]. The Canadian Network for Mood and Anxiety Treatments 2016 guidelines suggest rTMS as the first-line treatment for patients exhibiting treatment failure with at least one antidepressant [13]. However, in the current state, rTMS treatment limited feasibility that it requires daily treatment sessions for several weeks. Although rTMS treatment does not require considerable psychological or cognitive input from patients, as is the case for treatments such as psychotherapy or cognitive behavior therapy, it is burdensome in terms of physical effort.

Recently, it has been suggested that accelerated rTMS may provide a possibility for reducing treatment duration with effectiveness and safety comparable with those of conventional approaches. The accelerated rTMS protocol involves conducting more than one session per day, and reduces the number of visits required while providing the same total amount of stimuli. In previous retrospective and open-label studies, twice-daily rTMS treatment appears to achieve tolerability and effectiveness comparable with those of conventional once-daily rTMS treatment for MDD, while reducing the treatment duration to half
[14-16]. There are a few randomized controlled studies on accelerated rTMS treatment for depressive patients. One implied better effectiveness of accelerated rTMS than sham treatment in relieving the suicidal ideation and related distress of inpatients [17]. Another showed that the effectiveness of accelerated rTMS is comparable with that of conventional rTMS treatment in reducing depressive symptoms [18]. However, these studies also included bipolar depression or other disorders, such as post-traumatic stress disorder and mild traumatic brain injury, hard to guarantee homogeneity of subjects $[17,18]$. Only two randomized controlled study on accelerated rTMS treatment for patients with homogeneous diagnosis of MDD. Twice daily rTMS appeared to be effective and safe for MDD patients compared to sham [19]. Five daily rTMS treatment were applied to MDD patients and found the potential of fast clinical response compared to sham treatment in the other study [20]. These two studies compared only active accelerated rTMS to sham. There was no randomized controlled trial on unipolar depression comparing accelerated/conventional rTMS and sham treatments altogether.

Therefore, we investigated the effectiveness and safety of accelerated rTMS treatment for unipolar depression in comparison with conventional rTMS and sham treatments. Our primary hypothesis was that an accelerated rTMS protocol would result in a rapid improvement in depressive symptoms compared with a conventional rTMS protocol. In addition, we hypothesized that an accelerated rTMS would show effectiveness and tolerability comparable with conventional rTMS and better effectiveness than sham treatment.

\section{METHODS}

\section{Study Design}

The study was a three-arm single-blind RCT with randomization of patients into accelerated, conventional, or sham rTMS treatment protocols, conducted in two outpatient clinics of university hospitals in South Korea: Yonsei University Gangnam Severance Hospital (YUGSH) and Ajou University Hospital (AUH). Randomization was performed using an online randomizer (https://www. randomizer.org/). The clinicians who performed the treatment had knowledge of the treatment groups and the patients were aware of their own treatment protocol. Raters 
who assessed the symptoms were blind to the treatment groups. The patients were instructed not to disclose their treatment protocol to the raters before each assessment.

This study was approved by the institutional review board of Yonsei University Gangnam Severance Hospital (approval number: 3-2015-0178), and registered to the official website for clinical trial in Korea (https://cris.nih. go.kr/cris/index.jsp, registered number: KCT0000666). All the participants provided written informed consent after receiving a complete description of the study.

\section{Subjects}

We enrolled outpatients with MDD who were aged 2060 years; exhibited moderate-to-severe depression, with a Montgomery Asberg Depression Rating Scale score of $>$ 22; had exhibited the current episode for no more than 5 years; and were receiving one or two antidepressants, with stable doses, for 2 weeks. Patients were excluded if they had a contradiction to rTMS (such as the presence of metallic implants in the head, cochlear implants, cardiac pacemakers, or other implanted electronic devices); high risk of seizure (such as a personal or family history of seizure, brain tumor, or receiving drugs known to lower the seizure threshold); received rTMS treatment or electroconvulsive therapy; were found to exhibit another Diagnostic and Statistical Manual of Mental Disorders 4th edition (DSM-IV) Axis I psychiatric disorder; had a history of substance abuse or dependence; or had a past history of stroke, neurodegenerative disorder, or other major neurological or medical illness. Medication doses were kept unchanged during the trial.

\section{Assessment}

Demographic and clinical data were collected from psychiatric interview at baseline. Patients were diagnosed with MDD using the Structured Clinical Interview for DSM-IV. To investigate the time course of clinical effects on depressive symptoms, patients were rated using the self-report and clinician-rated versions of Korean Quick Inventory of Depressive Symptomatology (KQIDS-SR and KQIDS-C, respectively) at baseline, on day 3 , and at the end of weeks 3 and 6 (week 3 and week 6 , respectively). Patients were assessed using Clinical Global ImpressionSeverity (CGI-S) at the same time points as KQIDS-SR and KQIDS-C for general severity of illness and Clinical Global Impression-Improvement (CGI-I) on day 3, and at week 3 and week 6 for general improvement from baseline. For measuring safety, patients were assessed using the Frequency, Intensity, and Burden of Side Effects Rating (FIBSER) scale on day 3, and at week 3 and week 6.

\section{rTMS Treatment Session}

We conducted rTMS using the ALTMS $^{\circledR}$ stimulator (REMED Ltd., Seongnam, Korea; http://remed.kr/), consisting of a power generator with a control panel, figure of eight coil, and semi-reclined chair system with an arm for the stimulator coil. A subject's motor threshold was determined using the parameter estimation with sequential testing algorithm introduced in a previous study [21]. We used the sham stimulation protocol, with the same stimulation sound but no output through the coil from the generator.

We delivered rTMS to the left dorsolateral prefrontal cortex, which is defined as the position $5 \mathrm{~cm}$ anterior to the primary motor cortex area for the right abductor pollicis brevis muscle. Psychiatry residents who were trained for rTMS and not involved in the assessment of depressive symptoms and other outcome variables provided the treatment to the subjects. A single rTMS session was conducted with the figure of eight solid core coil at $110 \%$ motor threshold, $10 \mathrm{~Hz}, 5$-seconds duration, 25-seconds intertrain interval for 30 minutes (3,000 pulses) for the accelerated and conventional rTMS group. Five rTMS sessions were conducted per day for 3 days for the accelerated rTMS and sham-treatment group, but one rTMS session was conducted per day for 15 days, except weekend, for the conventional rTMS group.

\section{Statistical Analysis}

Descriptive analyses were conducted to compare baseline values among the treatment groups. Categorical variables were evaluated using chi-square test, and continuous variables using one-way analysis of variance. Concomitant psychotropic medications were analyzed using Fisher's exact test. Baseline to end-of-treatment changes in depressive symptoms and outcome variables regarding adverse events related to rTMS treatment were compared among the three treatment groups using linear mixed model for a repeated measures covariance pattern model with unstructured covariance within participants. Two fixed effects were included: one addressing the between group effect and one addressing the within time 


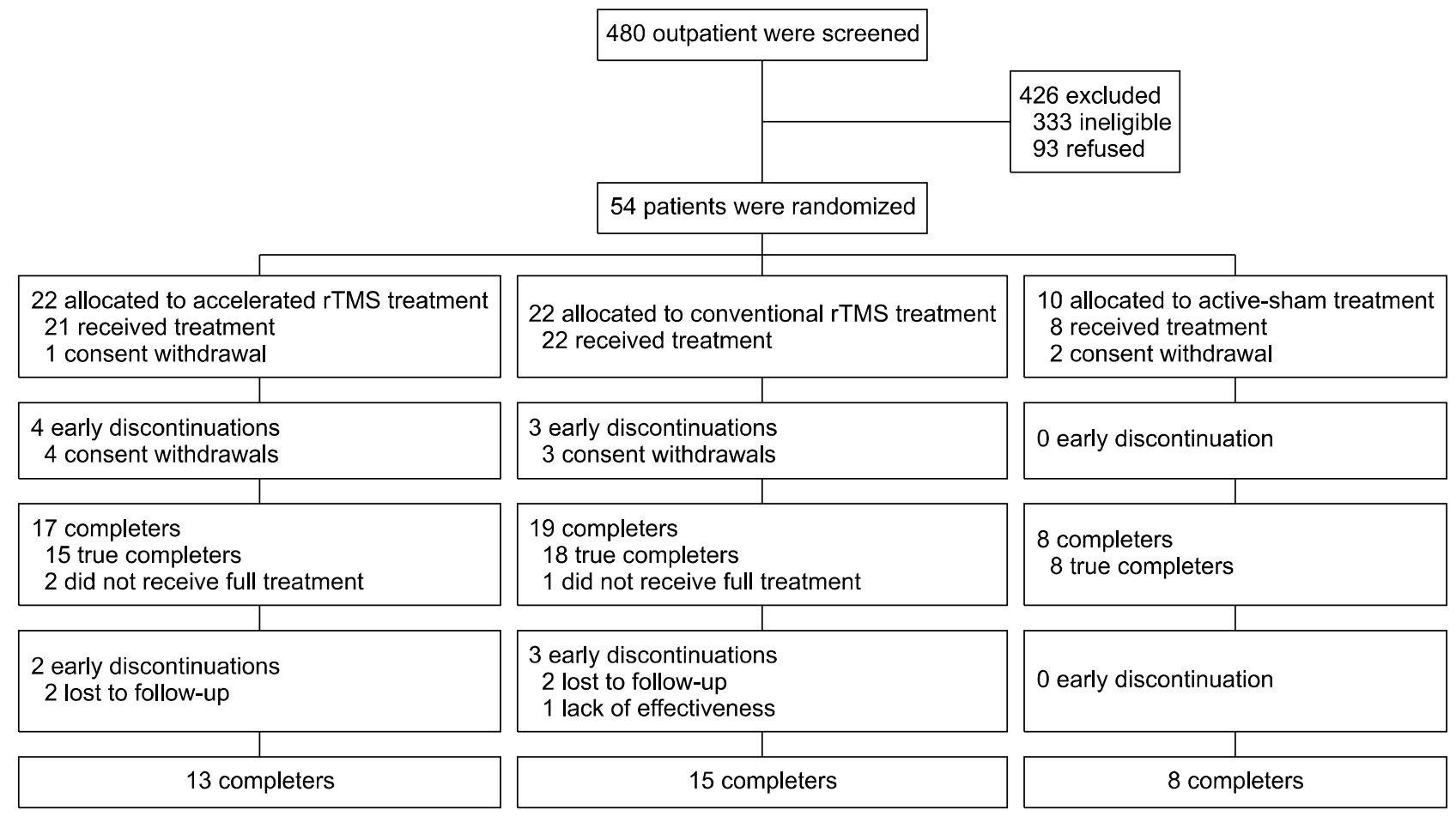

Fig. 1. CONSORT flowchart.

rTMS, repetitive transcranial magnetic stimulation.

effect. Post-hoc analyses were performed using independent $t$ tests at each time point of assessment between groups, with Bonferroni correction for multiple comparison. All statistical analyses were performed using the SAS software version 9.2 (SAS Inc., Cary, NC, USA). The threshold of statistical significance was set at 0.05 .

\section{RESULTS}

\section{Participants}

The study was conducted in accordance with the CONSORT guidelines as shown in Figure 1. Of the 480 patients screened (236 from YUGSH, 244 from AUH), 147 were found eligible ( 66 from YUGSH, 81 from $A U H$ ). Fifty-four patients consented and were recruited (24 from YUGSH, 30 from AUH). None were excluded at the baseline assessment. The 54 patients were randomized into the accelerated rTMS, conventional rTMS, and active sham groups at a ratio of 2:2:1 (accelerated rTMS: 22, conventional rTMS: 22, active sham: 10), considering lower treatment efficacy of sham treatment compared to active treatment from previous studies [10-12]. Three patients withdrew their consent before rTMS treatment (accelerated rTMS group: 1, active sham group: 2). Fiftyone patients started undergoing rTMS or sham treatment, and 36 patients completed treatment (accelerated rTMS: 13, conventional rTMS: 15, active sham: 8 ). Eight patients in the accelerated rTMS group and 7 patients in the conventional rTMS group dropped out, but none of the patients in the active sham group dropped out.

There were no differences in any demographic characteristic and baseline scores of any of the rating scales among the treatment groups (Table 1). The concomitant medications the patients were receiving did not show any differences among groups (Table 2).

\section{Primary Outcome}

In the KQIDS-SR score, as shown in Figure 2, there was a significant main effect of "time" $\left(\mathrm{F}_{3,47}=11.05, p<\right.$ $0.001)$, but no effect of "group" $\left(F_{2,47}=2.04, p=0.142\right)$, and a trend-level interaction effect of "group $\times$ time" $\left(\mathrm{F}_{6,47}=\right.$ 2.26, $p=0.053$ ). The KQIDS-SR scores at weeks 3 and 6 were significantly different between the accelerated rTMS and sham-treatment groups (week 3: $p=0.011$, week 6: $p=$ $0.027)$. The conventional rTMS group also showed significant differences in the change in KQIDS-SR scores 
Table 1. Demographic characteristics of the participants

\begin{tabular}{lccccc}
\hline \multicolumn{1}{c}{ Characteristics } & Accelerated rTMS $(\mathrm{n}=21)$ & Conventional rTMS $(\mathrm{n}=22)$ & Active sham $(\mathrm{n}=8)$ & $\mathrm{F}$ or $\left(\chi^{2}\right)$ & $p$ value \\
\hline Sex $($ male/female) & $4 / 17$ & $3 / 19$ & $2 / 6$ & 0.569 & 0.752 \\
Age $(\mathrm{yr})$ & $45.1 \pm 13.5$ & $43.9 \pm 10.9$ & $49.1 \pm 15.6$ & 0.484 & 0.619 \\
Years of education & $13.2 \pm 3.8$ & $13.1 \pm 3.9$ & $13.1 \pm 2.6$ & 0.004 & 0.996 \\
\hline
\end{tabular}

Data are presented as mean \pm standard deviation.

rTMS, repetitive transcranial magnetic stimulation.

Statistical significance of the differences among groups were tested usingone-way analysis of variance for continuous variables and chi-squared test for categorical variables.

Table 2. Concomitant psychotropic medications of participants

\begin{tabular}{lcccccc}
\hline \multicolumn{1}{c}{ Medication } & $\begin{array}{c}\text { Total } \\
(\mathrm{n}=51)\end{array}$ & $\begin{array}{c}\text { Accelerated rTMS } \\
(\mathrm{n}=21)\end{array}$ & $\begin{array}{c}\text { Conventional rTMS } \\
(\mathrm{n}=22)\end{array}$ & $\begin{array}{c}\text { Active sham } \\
(\mathrm{n}=8)\end{array}$ & $\chi^{2}$ & $p$ value \\
\hline SSRI & $22(43.1)$ & $9(42.9)$ & $12(54.5)$ & $1(12.5)$ & 4.114 & 0.128 \\
SNRI & $20(39.2)$ & $7(33.3)$ & $7(31.8)$ & $6(75.0)$ & 4.787 & 0.090 \\
Trazodone & $9(17.6)$ & $5(23.8)$ & $4(18.2)$ & $0(0.0)$ & 1.933 & 0.433 \\
Mirtazapine & $14(27.5)$ & $4(19.0)$ & $6(27.3)$ & $4(50.0)$ & 2.713 & 0.275 \\
Bupropion & $7(13.7)$ & $5(23.8)$ & $2(9.1)$ & $0(0.0)$ & 2.716 & 0.293 \\
Mood stabilizer & $7(13.7)$ & $2(9.5)$ & $4(18.2)$ & $1(12.5)$ & 0.794 & 0.858 \\
Olanzapine & $3(5.9)$ & $2(9.5)$ & $0(0.0)$ & $1(12.5)$ & 2.863 & 0.279 \\
Quetiapine & $17(33.3)$ & $8(38.1)$ & $7(31.8)$ & $2(25.0)$ & 0.492 & 0.854 \\
Aripiprazole & $12(23.5)$ & $7(33.3)$ & $2(9.1)$ & $3(37.5)$ & 4.790 & 0.081 \\
Benzodiazepine & $35(68.6)$ & $16(76.2)$ & $16(72.7)$ & $3(37.5)$ & 3.983 & 0.152 \\
\hline
\end{tabular}

Data are presented as number (\%).

rTMS, repetitive transcranial magnetic stimulation; SSRI, selective serotonin reuptake inhibitor; SNRI, serotonin norepinephrine reuptake inhibitor.

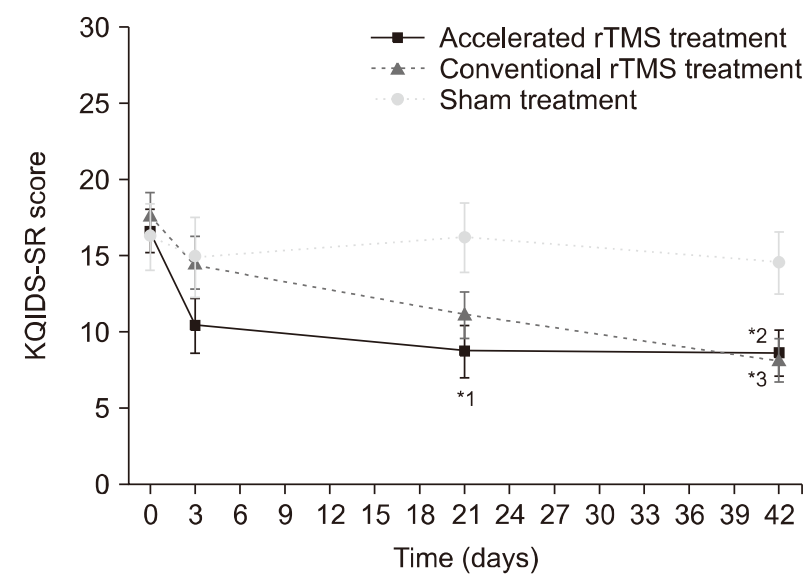

Fig. 2. The results of self-reported Korean Quick Inventory of Depressive Symptomatology (KQIDS-SR) across the study time points, analyzed using the linear mixed model 1: Three weeks after treatment, the KQIDS-SR score of the accelerated repetitive transcranial magnetic stimulation (rTMS) group was lower than that of the shamtreatment group. 2: Six weeks after treatment, the KQIDS-SR score of the accelerated rTMS group was lower than that of the sham-treatment group. 3: Six weeks after treatment, the KQIDS-SR score of the conventional rTMS group was lower than that of the sham-treatment group.

${ }^{*} p$ value $<0.05$ from baseline to week 6 compared with the sham-treatment group, but not in the change from baseline to week 3 (week 3: $p=0.069$, week $6: p=0.015$ ). There were no significant differences in the change in KQIDS-SR score between the accelerated and conventional rTMS groups at any time point (week 3: $p=0.210$, week 6: $p=0.840$ ).

The KQIDS-C score also showed similar results. There was a significant main effect of "time" $\left(\mathrm{F}_{3,47}=6.36, p<\right.$ $0.0001)$, but no effect of "group" $\left(F_{2,47}=2.28, p=0.114\right)$. There was only a trend-level "group $\times$ time" interaction effect $\left(\mathrm{F}_{6,47}=1.93, p=0.096\right)$. The KQIDS-C scores were significantly different at week 3 between the accelerated rTMS and sham-treatment groups, but not at week 6 (week 3: $p=0.011$, week 6: $p=0.154$ ). There was a trend-level, but not significant, difference in the KQIDS-C scores between the accelerated and conventional rTMS groups at week 3, but no such difference at week 6 (week 3: $p=0.096$, week 6: $p=0.798$ ). There were no significant differences between the conventional rTMS and sham-treatment groups (week 3: $p=0.188$, week 6: $p=$ $0.211)$. 
Table 3. Analysis of effectiveness in improvement of depressive symptoms using linear mixed model

\begin{tabular}{|c|c|c|c|c|c|c|}
\hline Variable & Time & $\begin{array}{c}\text { Accelerated } \\
\text { rTMS (A) }\end{array}$ & $\begin{array}{c}\text { Conventional } \\
\text { rTMS (B) }\end{array}$ & $\begin{array}{c}\text { Active Sham } \\
\text { (C) }\end{array}$ & $p$ value & Post-hoc \\
\hline \multirow[t]{4}{*}{ KQIDS-SR } & Baseline & $16.64(1.45)$ & $17.73(1.39)$ & $16.29(2.13)$ & Group: $p=0.142$ & (A) $<(\mathrm{C})$ at 3 weeks \\
\hline & 3 days & $10.42(1.80)$ & $14.57(1.70)$ & $14.89(2.60)$ & Time: $p<0.0001$ & $(\mathrm{~A})<(\mathrm{C})$ at 6 weeks \\
\hline & 3 weeks & $8.71(1.69)$ & $11.13(1.52)$ & $16.25(2.29)$ & Group $\times$ time: $p=0.053$ & $(\mathrm{~B})<(\mathrm{C})$ at 6 weeks \\
\hline & 6 weeks & $8.65(1.55)$ & $8.22(1.44)$ & $14.54(2.054)$ & & \\
\hline \multirow[t]{4}{*}{ KQIDS-C } & Baseline & $16.24(1.14)$ & $16.72(1.09)$ & $15.69(1.67)$ & Group: $p=0.114$ & (A) $<(\mathrm{C})$ at 3 weeks \\
\hline & 3 days & $9.97(1.72)$ & $13.81(1.61)$ & $14.89(2.46)$ & Time: $p<0.0001$ & \\
\hline & 3 weeks & $7.38(1.44)$ & $10.65(1.28)$ & $13.74(1.93)$ & Group $\times$ time: $p=0.096$ & \\
\hline & 6 weeks & $8.05(1.19)$ & $8.47(1.10)$ & $10.88(1.56)$ & & \\
\hline \multirow[t]{4}{*}{ CGI-S } & Baseline & $4.15(0.25)$ & $3.99(0.24)$ & $3.74(0.36)$ & Group: $p=0.444$ & (A) $<$ (B) at 3 weeks \\
\hline & 3 days & $3.27(0.36)$ & $3.55(0.34)$ & $3.56(0.51)$ & Time: $p<0.0001$ & (A) $<(\mathrm{C})$ at 6 weeks \\
\hline & 3 weeks & $2.40(0.32)$ & $3.32(0.29)$ & $3.36(0.44)$ & Group $\times$ time: $p=0.044$ & \\
\hline & 6 weeks & $2.54(0.22)$ & $2.89(0.21)$ & $3.41(0.29)$ & & \\
\hline \multirow[t]{3}{*}{ CGI-I } & 3 days & $2.72(0.33)$ & $2.85(0.31)$ & $3.56(0.47)$ & Group: $p=0.017$ & (A) $<$ (C) at 3 weeks \\
\hline & 3 weeks & $2.05(0.26)$ & $2.73(0.24)$ & $3.35(0.35)$ & Time: $p=0.24$ & (A) $<(\mathrm{C})$ at 6 weeks \\
\hline & 6 weeks & $2.04(0.27)$ & $2.70(0.25)$ & $3.10(0.35)$ & Group $\times$ time: $p=0.737$ & \\
\hline
\end{tabular}

Data are presented as mean (standard error).

rTMS, repetitive transcranial magnetic stimulation; KQIDS, Korean Quick Inventory of Depressive Symptomatology; -SR, self-reported; -C, clinician administered; CGI, Clinical Global Impression scale; -S, severity; -I, improvement.

Table 4. Frequency, Intensity, and Burden of Side Effects Rating scale score during treatment

\begin{tabular}{|c|c|c|c|c|c|c|}
\hline Variable & Time & $\begin{array}{c}\text { Accelerated } \\
\text { rTMS (A) }\end{array}$ & $\begin{array}{c}\text { Conventional } \\
\text { rTMS (B) }\end{array}$ & $\begin{array}{c}\text { Active sham } \\
\text { (C) }\end{array}$ & $p$ value & Post-hoc \\
\hline \multirow{3}{*}{$\begin{array}{c}\text { Frequency of } \\
\text { side effects }\end{array}$} & 3 days & $0.93(0.26)$ & $0.83(0.24)$ & $2(0.36)$ & Group: $p=0.021$ & (A) $<(\mathrm{C})$ at 3 day \\
\hline & 3 weeks & $0.59(0.14)$ & $0.78(0.13)$ & $1(0.19)$ & Time: $p=0.018$ & (B) $<$ (C)at 3 day \\
\hline & 6 weeks & $0.53(0.16)$ & $0.75(0.15)$ & $1.25(0.22)$ & Group $\times$ time: $p=0.238$ & $(\mathrm{~A})<(\mathrm{C})$ at 6 week \\
\hline \multirow{3}{*}{$\begin{array}{l}\text { Intensity of } \\
\text { side effects }\end{array}$} & 3 days & $1.2(0.28)$ & $1.06(0.26)$ & $1.63(0.39)$ & Group: $p=0.242$ & - \\
\hline & 3 weeks & $0.59(0.24)$ & $1.11(0.21)$ & $1(0.31)$ & Time: $p=0.186$ & \\
\hline & 6 weeks & $0.56(0.24)$ & $0.99(0.22)$ & $1.38(0.32)$ & Group $\times$ time: $p=0.333$ & \\
\hline \multirow{3}{*}{$\begin{array}{l}\text { Burden of } \\
\text { side effects }\end{array}$} & 3 days & $0.73(0.28)$ & $1.11(0.26)$ & $1.38(0.38)$ & Group: $p=0.310$ & - \\
\hline & 3 weeks & $0.67(0.28)$ & $0.94(0.25)$ & $1(0.38)$ & Time: $p=0.295$ & \\
\hline & 6 weeks & $0.64(0.21)$ & $0.69(0.20)$ & $1.38(0.28)$ & Group $\times$ time: $p=0.555$ & \\
\hline
\end{tabular}

Data are presented as mean (standard error).

rTMS, repetitive transcranial magnetic stimulation.

\section{Secondary Outcome}

As shown in Figure 3, there was a significant main effect of "time" $\left(\mathrm{F}_{3,46}=13.15, p<0.001\right)$ and "group $\times$ time" interaction $\left(\mathrm{F}_{6,46}=2.38, p=0.044\right)$ for the CGI-S scores, but no main effect of "group" $\left(\mathrm{F}_{2,47}=0.83, p=0.444\right)$. At week 3 , the CGI-S score of the accelerated rTMS group was significantly lower than that of the conventional rTMS group ( $p=0.038)$, and tended to be lower than that of the sham-treatment group ( $p=0.082)$. There was no difference between the conventional rTMS and sham-treatment groups ( $p=0.941)$. At week 6 , the CGI-S scores were significantly different only between the accelerated rTMS and sham-treatment groups $(p=0.022)$, not between the accelerated and conventional rTMS groups $(p=0.260)$ or between the conventional rTMS and sham-treatment groups $(p=0.148)$.

For the CGI-I score, there was a significant main effect of "group" $\left(F_{2,44}=4.45, p=0.017\right)$, but no effect of "time" $\left(\mathrm{F}_{2,44}=1.47, p=0.240\right)$ and "group $\times$ time" interaction $\left(\mathrm{F}_{4,44}=0.5, p=0.737\right)$. There were significant differences in the CGI-I score between the accelerated rTMS and sham-treatment groups (week 3: $p=0.005$, week $6: p=$ 0.020). There were trend-level differences between the accelerated and conventional rTMS groups at weeks 3 and 6 (week 3: $p=0.063$, week 6: $p=0.076$ ), but no significant differences between the conventional rTMS and 


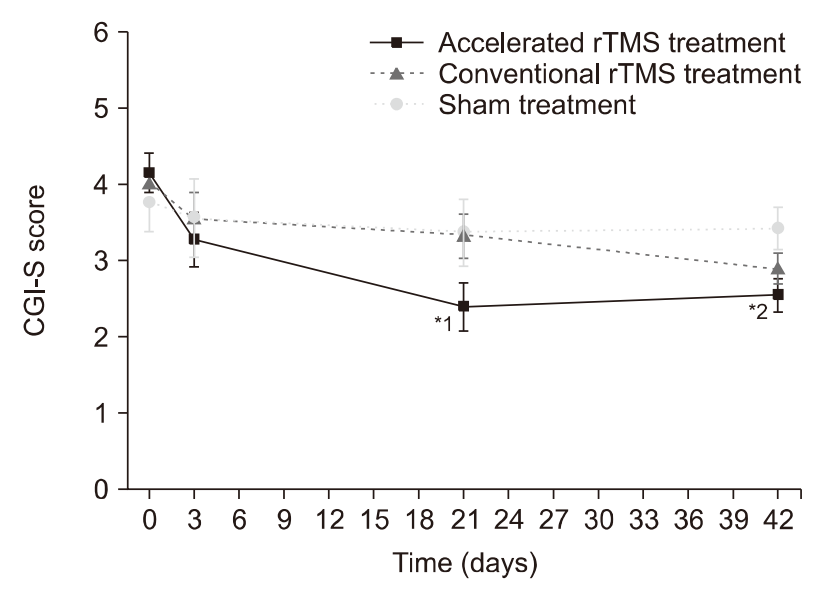

Fig. 3. Comparison of clinical global impression scale-severity (CGI-S) results using linear mixed model analysis 1: Three weeks after treatment, the CGI-S score of the accelerated repetitive transcranial magnetic stimulation (rTMS) group was lower than that of the conventional rTMS group. 2: Six weeks after treatment, the CGI-S score of the accelerated rTMS group was lower than that of the sham-treatment group.

${ }^{*} p$ value $<0.05$

sham-treatment groups (week 3: $p=0.149$, week 6: $p=$ 0.357) (Table 3).

\section{Tolerability of Treatment}

There were no serious adverse events in any of the treatment groups. In the frequency score of the FIBSER scale, there was a significant main effect of "group" $\left(\mathrm{F}_{2,38}=4.29\right.$, $p=0.021)$ and "time" $\left(\mathrm{F}_{2,38}=4.45, p=0.018\right)$, but no interaction effect of "group $\times$ time" $\left(\mathrm{F}_{4,38}=0.50, p=0.238\right)$. At day 3 , the FIBSER frequency scores of the accelerated and conventional rTMS groups were significantly lower than that of the sham-treatment group (accelerated rTMS: $p=0.022$, conventional rTMS: $p=0.010$ ), but there was no significant difference between the accelerated and conventional rTMS groups $(p=0.780)$. At week 3 , there were no significant differences in the frequency of side effects between the accelerated and conventional rTMS groups ( $p=0.349)$, between the accelerated rTMS and sham-treatment groups ( $p=0.098)$, or between the conventional rTMS and sham-treatment groups $(p=0.336)$. At week 6 , the frequency of side effects was lower in the accelerated rTMS group than in the sham group ( $p=$ $0.012)$, tended to be lower in the conventional rTMS group than in the sham group ( $p=0.065)$, and showed no significant difference between the accelerated and conventional rTMS groups $(p=0.336)$. There were no main effects of "group" $\left(\mathrm{F}_{2,38}=1.47, p=0.242\right)$ and "time" $\left(\mathrm{F}_{2,38}=\right.$ $1.76, p=0.186)$, nor interaction effect of "group $\times$ time" $\left(\mathrm{F}_{4,38}=1.19, p=0.333\right)$, in intensity score of the FIBSER scale. There were also no main effects of "group" $\left(\mathrm{F}_{2,38}=\right.$ $1.21, p=0.310)$ and "time" $\left(F_{2,38}=1.26, p=0.295\right)$, nor interaction effect of "group $\times$ time" $\left(\mathrm{F}_{4,38}=0.76, p=\right.$ 0.555 ) in the burden score of the FIBSER scale (Table 4).

\section{DISCUSSION}

In this study, the accelerated rTMS treatment group showed favorable effectiveness and tolerability compared with the sham treatment group, and comparable effectiveness and safety profile with the conventional rTMS treatment group in patients with MDD.

No significant improvement in depressive symptoms was observed in any scale of KQIDS and CGI 3 days after completion of the accelerated rTMS treatment compared with sham or conventional rTMS treatment. In the previous study, a dramatic improvement in the suicidal ideation was observed on the first day of the treatment, but it did not differ from that of sham on day 3 , after completion of the treatment protocol [17].

In the present study, the improvement of depressive symptoms was noted at week 3. Both KQIDS-SR and KQIDS-C scores at week 3 were lower in the accelerated rTMS group than in the sham-treatment group. Although the difference in the improvement of depressive symptoms between the accelerated rTMS and the conventional rTMS groups was not statistically significant, considering the lack of difference between the conventional rTMS and sham-treatment groups, the accelerated rTMS protocol has potential to be beneficial for rapid improvement of depressive symptoms compared with the conventional method. This is supported by the result that the accelerated rTMS group scored lower that the conventional rTMS group at week 3 in CGI-S, although this scale does not selectively assess depressive symptoms. A delay in the improvement of depressive symptoms becomes evident in the accelerated rTMS treatment; this might be related to the up-to-two weeks of time required for antidepressants to act. It is known that rTMS contributes to synaptic plasticity at various levels, such as cellular $\mathrm{Ca}^{2+}$ dynamics, modulation in the levels and the functions of neurotransmitters and neurotrophic factors, orchestration of the neuroendocrine and inflammation systems, and orches- 
tration of the glial network [22,23]. As with antidepressants, rTMS is expected to exert a therapeutic effect through synaptic plasticity, but direct evidence is insufficient because studies on this neurobiological basis have not yet been conducted on patients with depression. A previous study on patients with treatment-resistant depression attempted to explore the effect of accelerated rTMS on the levels of neurotransmitters, such as glutamate and $\gamma$-aminobutyric acid, using proton magnetic resonance spectroscopy, but the results obtained were negative [24]. More future studies are needed in this area to clarify the neurobiological mechanism of the therapeutic effect of accelerated rTMS on depression.

In the present study, at week 6, the accelerated rTMS group showed greater improvement in the KQIDS-SR, CGI-S, and CGI-I scores than the sham-treatment group. The score of KQIDS-SR, which contains more specific questions on depressive symptoms, significantly improved in the accelerated rTMS group compared with the sham-treatment group, but KQIDS-C score did not differ. Due to the difference in treatment duration, blinding was possible for the accelerated rTMS and sham-treatment groups, but not for the conventional rTMS group. Patients were not informed whether they were assigned to the accelerated rTMS or sham-treatment group. Although this was a single-blind study, considering that partial blinding was possible, the observed improvement would not have occurred due to placebo effect. The KQIDS-SR and KQIDS-C each uniquely contribute to the prediction of treatment outcome [25]. A balance between these measures is required to determine the extent of improvement in depressive symptoms. In addition, since the improvement of both CGI-S and CGI-I scores, which were also provided by clinicians, was more evident in the accelerated rTMS group than in the sham-treatment group, it is reasonable to conclude that there was a significant symptom improvement at week 6 . This follow-up result is different from those of a previous RCT in which there was no difference between the accelerated rTMS and sham-treatment groups after 3 and 6 months [17]. This discrepancy could have stemmed from the differences in the follow-up time points difference between the two studies. However, there is only one RCT comparing accelerated rTMS to sham treatment. Therefore, further investigation, with more RCTs with larger sample size, is required. In the present study, there was no significant difference in the
KQIDS-SR, KQIDS-C, CGI-S, and CGI-I scores between accelerated and conventional rTMS treatments at week 6 , which was the last time point for symptom assessment. This result is concordant with those of previous studies which showed that twice-daily rTMS achieves results comparable with once-daily (conventional) rTMS in an open-label [14], or to an even more favorable extent, at a trend-level, in a retrospective study [15], and an RCT that showed no significant reduction of depressive symptoms or remission or response rates between the accelerated and standard (conventional) rTMS groups [18].

More interestingly, at day 3 and week 6 , the accelerated rTMS group reported a lower frequency of side effects than the sham-treatment group. Considering that there were 7 early discontinuation cases each in the accelerated and conventional rTMS groups, but none in the sham-treatment group, patients who experienced more side effects in the rTMS groups could have been excluded in the final analysis. Previous studies have reported that the pain in the rTMS site or headache may be increased in accelerated rTMS $[17,18]$. In the present study, we did not question the patients regarding adverse events using specific terms such as "site pain" or "headache," and this difference in the methodology of questioning could have caused the observed differences. Further, unlike previous studies which examined side effects after each session, the first side effect inquiry was conducted on day 3 after completion of accelerated rTMS treatment. Discomfort due to accelerated rTMS tended to be relieved by day 3 [17]; therefore, it is possible that the initial side effect had already reduced when the side effect questionnaire was administered. Nevertheless, the frequency of side effects reported was rather less in the accelerated rTMS group than in the sham-treatment group. This could be attributed to the inability to distinguish treatment-relevant side effects and various somatic symptoms due to depression itself. It is suggested that depressive symptoms rapidly improved in the accelerated rTMS group, and therefore, the somatic discomfort caused by depression in this group would be less. A more accurate measurement of side effects using a structured questionnaire or interview, with clinicians assessing the relevance of the side effects to the treatment, is necessary.

This is the first three-arm randomized controlled study comparing the efficiency and tolerability of accelerated rTMS with those of conventional rTMS and sham 
treatment. Accelerated rTMS appears to be as effective and safe as conventional rTMS. Early discontinuation rate and scores of the frequency, intensity, and burden of side effects were also not significantly different between the accelerated and conventional rTMS groups. This result indicates that intensive treatment for 3 days using the accelerated rTMS protocol can achieve the same level of therapeutic effect as conventional rTMS. Accelerated rTMS would free patients from the hassle of visiting the hospital daily for 3 weeks to undergo treatment. Even the patients with MDD who are hardly expected to visit psychiatric clinics due to severe depressive symptoms or long distance from where their residence can benefit from accelerated rTMS via short-term hospitalization for 3 days. In the aspect of accessibility, the findings from this study appear to be encouraging. Future studies on inpatients with MDD exhibiting more severe symptoms, such as high risk of suicide, are required to validate and conclusively demonstrate the advantages of accelerated rTMS. A previous pilot study on inpatients with suicidal ideation has not yielded clear results on the effectiveness of rTMS [17], but there remains a lack of sufficient and qualified evidences.

In spite of several strengths, the present study also has some critical and inevitable limitations. First, the difference in treatment duration between the accelerated and the conventional rTMS protocols led to incomplete blinding of the patients. Therefore, a completely double-blind RCT was not possible. Since the conventional rTMS group was treated for 3 weeks and the sham-treatment and accelerated rTMS groups were treated for only 3 days, the patients would have at least realized whether they were in the conventional or the sham-treatment/accelerated group. In order to overcome this limitation, the patients should have been assigned to active accelerated rTMS + sham conventional rTMS, sham accelerated rTMS + active conventional rTMS, and sham accelerated rTMS + sham accelerated rTMS groups. This would have required 3.5 weeks to complete the treatment protocol. Moreover, only a few patients would agree to be enrolled in a clinical study after being informed that they may only receive sham treatment despite visiting the clinic for 18 days. In this regard, the design of our study can be considered the most economical for conducting an RCT comparing the accelerated with conventional rTMS groups. In addition, as mentioned above, blinding for the accelerated rTMS and sham-treatment groups was retained appropriately in the present study. Second, as noted above, the scale used for assessing adverse effects in the present study was not very helpful in detecting the effects specifically associated with rTMS treatment. Patients with depression may misidentify various somatic symptoms originating from depression itself as being caused by rTMS treatment. Using a measure that allows the clinician to objectively assess whether the adverse events are directly related to the treatment would have been more helpful in accurately assessing the safety. Third, this study was conducted on small number of subjects, especially in the active sham group, from only two institutions. Half number of the active treatment groups were assigned as active sham group based on the previous evidences of low efficacy of sham-treatment. According to our finding that the KIDS-C and CGI-S scores showed no significant difference between conventional rTMS and active sham group, it cannot be guaranteed that the sham-treatment is less effective than conventional rTMS treatment. The CGI-I score showed only a trend-level difference, but it would not be sufficient to be reflected to the significant difference in the CGI-S scores. Larger sample size with increased ratio of the active sham group to the level of other treatment groups from several more centers in Korea or abroad is required to generalize our findings.

The present study shows that there is no significant final difference in the effectiveness and safety of accelerated and conventional rTMS treatments for depressive symptoms. With the merit of resulting in early improvement of depressive symptoms, the accelerated rTMS protocol deserves more attention. In summary, accelerated rTMS treatment could be a viable treatment option for MDD, with shortened treatment duration. Further studies with large sample size and long-term follow-up are recommended.

\section{acknowledgments}

This clinical trial is based upon work supported by two grants of the Korean Health and Technology R\& D project, Ministry of Health\& Welfare (grant number: A1020651112-1500100) and the Ministry of Trade, Industry \& Energy (MOTIE) under the Industrial Technology Innovation Program (grant number: 10049734), Republic of Korea. The authors would also like to show our gratitude to the people whose participation made this study possible. Dongsoo Kim, Kyeongmin Cha, Gyu Ha Kang, Tae-Hoon 
Kwon, Sunghyun Park, Hyojin Han and Kyeongseon Yun performed rTMS procedure; Soyoung Sung, Woonsook Jeon and Seulbit Pi measured psychological data; and Min-Kyung Kim outlined the draft.

\section{- Conflicts of Interest}

No potential conflict of interest relevant to this article was reported.

\section{Author Contributions}

Conceptualization: Jeong-Ho Seok, Jai-Sung Noh. Data acquisition: Sang Joon Son, Mi Jang, Byung-Hoon Kim, Seok Joo Hong, Lina Seo, and Sun-Woo Choi. Formal analysis: Soo-Jeong Kim, Jeong-Ho Seok. Funding: Jeong-Ho Seok, Jai Sung Noh. Supervision: Jeong-Ho Seok, Jai Sung Noh. Writing - original draft: Soo-Jeong Kim, Jeong-Ho Seok. Writing - review \& editing: Soo-Jeong Kim, Jeong-Ho Seok.

\section{ORCID}

Soo-Jeong Kim https://orcid.org/0000-0001-9065-7492

Sang Joon Son https://orcid.org/0000-0001-7434-7996 Mi Jang https://orcid.org/0000-0003-4558-1171

Byung-Hoon Kim https://orcid.org/0000-0003-2501-038X Seok Joo Hong https://orcid.org/0000-0003-3191-7287 Lina Seo

Sun-Woo Choi https://orcid.org/0000-0002-2681-4518 Jeong-Ho Seok https://orcid.org/0000-0003-0755-9387 Jai Sung Noh https://orcid.org/0000-0002-9402-7591 https://orcid.org/0000-0002-9610-0756

\section{REFERENCES}

1. World Health Organization. Preventing suicide: a global imperative. Geneva:World Health Organization;2014.

2. World Health Organization. Mental health action plan 2013-2020. Geneva:World Health Organization;2013. p.48.

3. Kessler RC, Berglund P, Demler O, Jin R, Koretz D, Merikangas KR, et al.; National Comorbidity Survey Replication. The epidemiology of major depressive disorder: results from the National Comorbidity Survey Replication (NCS-R). JAMA 2003;289:3095-3105.

4. Srisurapanont M, Hong JP, Tian-Mei S, Hatim A, Liu CY, Udomratn P, et al.; MD RAN. Clinical features of depression in Asia: results of a large prospective, cross-sectional study. Asia Pac Psychiatry 2013;5:259-267.

5. Whiteford HA, Ferrari AJ, Degenhardt L, Feigin V, Vos T. The global burden of mental, neurological and substance use disorders: an analysis from the Global Burden of Disease Study 2010. PLoS One 2015;10:e0116820.
6. Lin CH, Yen YC, Chen MC, Chen CC. Depression and pain impair daily functioning and quality of life in patients with major depressive disorder. J Affect Disord 2014;166:173-178.

7. Sinyor M, Schaffer A, Levitt A. The sequenced treatment alternatives to relieve depression (STAR*D) trial: a review. Can J Psychiatry 2010;55:126-135.

8. Sackeim HA, Prudic J, Fuller R, Keilp J, Lavori PW, Olfson M. The cognitive effects of electroconvulsive therapy in community settings. Neuropsychopharmacology 2007;32:244-254.

9. Martin DM, McClintock SM, Forster J, Loo CK. Does therapeutic repetitive transcranial magnetic stimulation cause cognitive enhancing effects in patients with neuropsychiatric conditions? A systematic review and meta-analysis of randomised controlled trials. Neuropsychol Rev 2016;26:295-309.

10. Brunoni AR, Chaimani A, Moffa AH, Razza LB, Gattaz WF, Daskalakis ZJ, et al. Repetitive transcranial magnetic stimulation for the acute treatment of major depressive episodes: a systematic review with network meta-analysis. JAMA Psychiatry 2017;74:143-152.

11. Gaynes BN, Lloyd SW, Lux L, Gartlehner G, Hansen RA, Brode $\mathrm{S}$, et al. Repetitive transcranial magnetic stimulation for treatment-resistant depression: a systematic review and meta-analysis. J Clin Psychiatry 2014;75:477-489; quiz 489.

12. Liu B, Zhang Y, Zhang L, Li L. Repetitive transcranial magnetic stimulation as an augmentative strategy for treatment-resistant depression, a meta-analysis of randomized, double-blind and sham-controlled study. BMC Psychiatry 2014;14:342.

13. Milev RV, Giacobbe $P$, Kennedy SH, Blumberger DM, Daskalakis ZJ, Downar J, et al.; CANMAT Depression Work Group. Canadian Network for Mood and Anxiety Treatments (CANMAT) 2016 Clinical Guidelines for the Management of Adults with Major Depressive Disorder: section 4. neurostimulation treatments. Can J Psychiatry 2016;61:561-575.

14. Schulze L, Feffer K, Lozano C, Giacobbe P, Daskalakis ZJ, Blumberger DM, et al. Number of pulses or number of sessions? An open-label study of trajectories of improvement for once-vs. twice-daily dorsomedial prefrontal rTMS in major depression. Brain Stimul 2018;11:327-336.

15. Modirrousta M, Meek BP, Wikstrom SL. Efficacy of twice-daily vs once-daily sessions of repetitive transcranial magnetic stimulation in the treatment of major depressive disorder: a retrospective study. Neuropsychiatr Dis Treat 2018;14:309-316.

16. McGirr A, Van den Eynde F, Tovar-Perdomo S, Fleck MP, Berlim MT. Effectiveness and acceptability of accelerated repetitive transcranial magnetic stimulation (rTMS) for treatment-resistant major depressive disorder: an open label trial. J Affect Disord 2015;173:216-220.

17. George MS, Raman R, Benedek DM, Pelic CG, Grammer GG, Stokes KT, et al. A two-site pilot randomized 3 day trial of high dose left prefrontal repetitive transcranial magnetic stimulation (rTMS) for suicidal inpatients. Brain Stimul 2014;7: 421-431.

18. Fitzgerald PB, Hoy KE, Elliot D, Susan McQueen RN, 
Wambeek LE, Daskalakis ZJ. Accelerated repetitive transcranial magnetic stimulation in the treatment of depression. Neuropsychopharmacology 2018:43:1565-1572.

19. Loo CK, Mitchell PB, McFarquhar TF, Malhi GS, Sachdev PS. A sham-controlled trial of the efficacy and safety of twice-daily rTMS in major depression. Psychol Med 2007;37:341-349.

20. Baeken C, Vanderhasselt MA, Remue J, Herremans S, Vanderbruggen $\mathrm{N}$, Zeeuws D, et al. Intensive HF-rTMS treatment in refractory medication-resistant unipolar depressed patients. J Affect Disord 2013;151:625-631.

21. Mishory A, Molnar C, Koola J, Li X, Kozel FA, Myrick H, et al. The maximum-likelihood strategy for determining transcranial magnetic stimulation motor threshold, using parameter estimation by sequential testing is faster than conventional methods with similar precision. J ECT 2004;20:160-165.
22. Chervyakov AV, Chernyavsky AY, Sinitsyn DO, Piradov MA. Possible mechanisms underlying the therapeutic effects of transcranial magnetic stimulation. Front Hum Neurosci 2015; 9:303.

23. Cirillo G, Di Pino G, Capone F, Ranieri F, Florio L, Todisco V, et al. Neurobiological after-effects of non-invasive brain stimulation. Brain Stimul 2017;10:1-18.

24. Baeken C, Lefaucheur JP, Van Schuerbeek P. The impact of accelerated high frequency $r$ TMS on brain neurochemicals in treatment-resistant depression: insights from $1 \mathrm{HMR}$ spectroscopy. Clin Neurophysiol 2017;128:1664-1672.

25. Uher R, Perlis RH, Placentino A, Dernovšek MZ, Henigsberg $\mathrm{N}$, Mors $\mathrm{O}$, et al. Self-report and clinician-rated measures of depression severity: can one replace the other? Depress Anxiety 2012;29:1043-1049. 